\title{
Eventualidades que intervienen en el abandono escolar de los estudiantes de la Licenciatura en Idiomas: Caso DAEA, UJAT
}

\author{
Eventualities that intervene in the drop-out of students of the Bachelor of Languages: \\ Case DAEA, UJAT \\ Doris L. B. Dzib Moo ${ }^{a}$, Rocio Hernández Hernández ${ }^{b}$, María T. Camacho Pérez $^{c}$
}

\begin{abstract}
:
The partial results of research, presented in this article, are intended to identify how some indicators behave that could influence the dropout of students from the Bachelor of Languages at the Universidad Juárez Autónoma de Tabasco. This is due to the fact that in the last five years, there has been an increase in the dropout rate in the last semesters of said Degree. This, despite the fact that the career has high job expectations at national and international level. For this reason, with the research report presented, it is intended to present a general panorama that allows for reflection and analysis in the search for possible solutions to this problem. The article is structured in four parts: the first includes contextual and conceptual references on the teaching of languages, their methods and the principles of learning. The second exposes the problem to be investigated and its objectives, as well as the methodology used. The third part presents the results of the application of a questionnaire aimed at students of the second semester of this career. We conclude by reflecting on the possibilities offered by the results to attend to the eventualities that could affect the drop-out in this degree.
\end{abstract}

Keywords:

School dropout. Learning styles. Teaching of a foreign language. University students

\section{Resumen:}

Los resultados parciales de investigación, que se presentan en este artículo, tienen como finalidad identificar cómo se comportan algunos indicadores que podrían influir en el abandono escolar de los estudiantes de la Licenciatura en Idiomas de la Universidad Juárez Autónoma de Tabasco. Esto debido a que en el último quinquenio, ha habido aumento del índice de abandono escolar en los últimos semestres de dicha Licenciatura. Esto, a pesar de que la carrera cuenta con altas expectativas laborales, tanto nacionales como internacionales. Por ello, con el reporte de investigación que se presenta se pretende dar a conocer un panorama general que permita la reflexión y el análisis en la búsqueda de posibles soluciones a esta problemática. El artículo se estructura en cuatro partes: la primera incluye referentes contextuales y conceptuales sobre la enseñanza de lenguas, sus métodos y los principios de aprendizaje. La segunda expone el problema a investigar y sus objetivos, así como la metodología utilizada. La tercera parte presenta los resultados de aplicación de un cuestionario dirigido a estudiantes de segundo semestre de dicha carrera. Concluimos reflexionando sobre las posibilidades que ofrecen los resultados para atender las eventualidades que pudieran incidir en el abandono escolar en esta licenciatura.

\section{Palabras Clave:}

Abandono escolar. Estilos de aprendizaje. Enseñanza de lengua extranjera. Estudiantes universitarios

\footnotetext{
a Profesora investigadora de la División Académica de Educación y Artes de la Universidad Juárez Autónoma de Tabasco.

Villahermosa, Tabasco, México. https://orcid.org/0000-0002-6559-0879 Email: dorisdzib@ hotmail.com

b Profesora Investigadora de la División Académica de Educación y Artes de la Universidad Juárez Autónoma de Tabasco. Villahermosa, Tabasco, México. Email: rocahdhd@hotmail.com

c Profesora Investigadora de la División Académica de Educación y Artes de la Universidad Juárez Autónoma de Tabasco. Villahermosa, Tabasco, México. Email: maría.camacho@ujat.mx
} 


\section{Introducción}

La presente investigación fue motivada en la intención de identificar las eventualidades que han propiciado el aumento en el índice del abandono escolar de los estudiantes de la Licenciatura en idiomas de la División Académica de Educación y Artes de la Universidad Juárez Autónoma de Tabasco.

Como es sabido en la actualidad el dominio del idioma inglés es de carácter indispensable, por ser catalogada como una lengua comercial e internacional por excelencia, ya que tiene una diversidad amplia para ser utilizada en una cantidad cada vez mayor de países y en el propio territorio mexicano. Por otro lado, se le ha catalogado como una herramienta que posibilita la activación de los dos hemisferios del cerebro, como prevención de la demencia, fomenta la memoria y la concentración.

Para muchas personas, es atractivo el dominio del idioma inglés como segunda lengua, pero la aplicación intrínseca de aprender a través de la música, películas, y video-juegos, entre otras posibilidades, propicia que el aprendizaje sea más fácil, es por eso que a partir de esas prácticas cotidianas, se tienen posibilidades de incursionar en el mercado laboral de manera favorable, en donde es requisito indispensable el dominio total del idioma inglés.

Desde dichas reflexiones, se presenta este reporte de investigación, que dividimos cuatro partes. La primera presenta algunos referentes históricos que señalan la importancia de la enseñanza de las lenguas y cómo se van definiendo sus métodos de enseñanza, además de incluir, como base conceptual, los principios que están presentes en el aprendizaje de las lenguas. En el segundo apartado se expone el problema a investigar con sus respectivos objetivos y metodología, para después, en la tercera parte, presentar los resultados de la aplicación de una encuesta a los estudiantes y de ahí derivar conclusiones.

\section{Marco contextual}

\section{Historia de la enseñanza del idioma inglés}

La historia de la enseñanza de una lengua extranjera siempre ha sido un tema en discusión desde diferentes perspectivas y en algunos casos señalando un método de enseñanza en específico de acuerdo con la experiencia o solo desde la teoría. En las últimas décadas, se puede percibir como hay mejoras en los métodos de enseñanza, y en consecuencia cambios en el dominio del idioma día con día.
De acuerdo con Kelly (1969) y Howatt (1984), existen muchos referentes sobre la enseñanza del idioma que no eran nuevas, por ejemplo, consideran que un $60 \%$ de la población en el mundo es multilingüe, por lo que el manejo de diversidad de idiomas se da en contextos diversos. Hoy día el idioma inglés es el más estudiado, desde hace aproximadamente unos 500 años. El más aprendido fue el latín, debido a que era el lenguaje más demandado en diferentes áreas como: el comercio, la religión y el gobierno en el mundo occidental.

En el siglo XVI, debido al resultado de los cambios políticos en Europa, el inglés, francés e italiano comenzaron a tener ventajas y al mismo tiempo el latín comenzó a dejarse de hablar y escribir. A raíz de esto el latín comenzó a decaer y dejar de ser una lengua viva y paso a tener otra función, principalmente en las escuelas. En consecuencia, en los siglos XVI, XVII y XVIII, el estudio del latín clásico, análisis de la gramática y la retórica, dio origen al modelo del estudio de la lengua extrajera, es así como comienza el estudio y la importancia de la enseñanza de las lenguas extranjeras. En Inglaterra, los niños comenzaron con el estudio de la gramática en los siglos XVI, XVII y XVIII, es así como se comienza a impartir una pequeña introducción a la gramática del latín. Revisando sus reglas gramaticales, declinaciones, conjugaciones, traducciones y práctica de ejemplos de oraciones escritas y en algunas ocasiones, con el uso paralelo bilingüe de textos y diálogos. Esto propició el establecimiento de una competencia básica, que los estudiantes fueran introducidos en el estudio avanzado de la gramática y la retórica.

Una vez que el latín había dejado de ser un vehículo normal de comunicación, y que fue reemplazado como tal por las lenguas vernáculas, entonces rápidamente se convirtió en una "gimnasia mental"; el lenguaje supremamente "muerto", se convirtió en un estudio disciplinado y sistemático de las diversas disciplinas, siendo indispensable como base de todas las formas de la educación superior (Mallison, en Titone 1968: 26).

Para el siglo XIX, el enfoque que se basaba en el estudio del latín había llegado al estándar en el estudio de las lenguas extranjeras en las escuelas, utilizando el típico libro de texto que solo consistía en capítulos o lecciones construidas con base en aspectos de gramática. Cada punto de gramática era enunciado, además de las reglas, y solo eran ilustradas por ejemplos de oraciones. Para el siglo XIX los libros de texto copilaban de igual forma la codificación de una lengua extranjera, las reglas de morfología y sintaxis y eran explicadas y eventualmente memorizadas.

De acuerdo con Titone (1968: 27), la situación anterior dio origen a diferentes libros, en los que estos se dividían en dos partes: los pasos gramaticales y enseguida se comenzaba a dar frases sencillas, por ejemplo: "Él tiene 
un libro y un perro". A raíz de esto se da comienzo al enfoque de la enseñanza de una lengua extranjera, la cual se identifica con el método "Gramar-traslation".

Grammar-traslation fue conocido, primeramente, en Estados Unidos de América como Prussian Method, las principales características de este método son: tener un objetivo al momento de aprender la lengua extranjera, de acuerdo con lo que se vaya adquiriendo, conforme se vaya leyendo la literatura o de acuerdo al orden de beneficio de la disciplina mental y el desarrollo intelectual.

Grammar-traslation se enfoca esencialmente en el análisis de las reglas de gramática, este método de la enseñanza de una lengua extranjera predominó en Europa desde los 1840 hasta 1940, y a pesar de las modificaciones, aún se continúa utilizando en algunas partes del mundo. A pesar de todos los cambios e innovaciones que se han dado, se siguen realizando trabajos constantes con este enfoque pero a su vez, han surgido propuestas que han propiciado innovaciones en la enseñanza de una lengua extrajera.

El estudio de la lengua hablada, el entrenamiento de la fonética en vías de que se vuelva habito y poder obtener una buena pronunciación, el uso de conversión de textos y diálogos a modo de inducir a conversiones de frases y dialectos, son algunas de las estrategias para el acceso y dominio de la lengua. Dos más de ellas, son: una inducción al enfoque de cómo enseñar gramática, así como la enseñanza de nuevos significados por medio de la asociación entre los objetivos del idioma y la lengua madre. No obstante, surgieron controversias referentes a cuál debía de ser la mejor forma para la enseñanza de lenguas extranjeras, por lo que se generaron diversas propuestas.

Una de las propuestas que emergieron fue la de Sweet (1845-1912), Wilhelm Viëtor (1850-1918), la cual fue denominada por los miembros de la reforma del movimiento como método natural. Conforme a su desarrollo llegó a ser conocido como hasta la fecha se le conoce "método directo". Jack C. Richards (2003), argumenta que el método natural o método directo de una lengua extranjera se puede enseñar sin traducción o sin el uso de la lengua materna del estudiante, siempre y cuando si el significado fue transmitido directamente a través de la demostración y la acción. El método directo fue introducido en Francia y Alemania, y fue oficialmente aprobado en ambos países a finales del siglo XIX, y se hizo ampliamente conocido en los Estados Unidos a través de Sauver y Maximilian Berlitz en las escuelas de éxito comercial.

De acuerdo con H. Douglas Brown (1994), en el siglo XX, fue cuando comenzaron los comentarios y series de debates en relación a los diferentes métodos de enseñanza, además de infinidad de enfoques, métodos y supuestos, es a esto lo que se le conoce como "la era de métodos". El periodo más significante en la historia de enfoques y métodos de enseñanzas de lenguas fue entre los años cincuenta y ochenta del siglo XX. Entre los cincuenta y sesenta fue donde se vio el surgimiento del método audio lingual y el método de la situación, que se sustituyó por el enfoque comunicativo, los cuales se encuentran en uso en la actualidad.

A pesar de los cambios de estatus de los diferentes enfoques y métodos en la enseñanza del idioma, los estudios tanto pasados como presentes sobre los métodos de enseñanza, continúan de forma significativa como componentes esenciales en la preparación de los profesores de programas de enseñanza de lenguas en la actualidad.

Además de lo anterior, existen una serie de principios que están presentes en el proceso formativo de los estudiantes de idiomas por lo que es necesario hacer referencia a cuáles son éstos en lo particular, por ello estos se desarrollan a continuación.

\section{Marco conceptual}

\section{El Principio de la motivación intrínseca}

De acuerdo con José Bernardo Carrasco y Basterretche (2004), el principio de la motivación intrínseca son las recompensas más poderosas, y son aquellas que están motivadas intrínsecamente en el estudiante. Debido a que el comportamiento se deriva de necesidades, deseos o deseos dentro de uno mismo, el comportamiento en sí es auto-gratificante, por lo tanto, la recompensa no administrada externamente es necesaria en lo absoluto.

La motivación intrínseca se puede apreciar cuando el estudiante es testigo de una actividad, que por el simple placer de realizarla, sin ninguna instrucción por parte de otra persona, la puede realizar. Por ejemplo, un pasatiempo es un claro ejemplo de esta motivación, al igual que la sensación de placer o la auto-superación o en su caso la sensación de éxito. Si todos los estudiantes estuviesen motivados intrínsecamente para realizar todas las tareas en el aula, ni siquiera podríamos necesitar profesores, se puede realizar un gran servicio a los estudiantes y al proceso general de aprendizaje, considerando cuidadosamente, en primer lugar, cuáles son los motivos intrínsecos de los estudiantes y, posteriormente, al diseñar tareas en el aula que se alimenten en esas unidades intrínsecas.

Las técnicas de aula tienen muchas probabilidades de éxito si son auto-gratificantes en la percepción de los estudiantes; ellos realizarán la tarea porque sería divertido para ellos, además de resultar interesante, útil y en algunos casos difícil, y las realizarían no porque el 
profesor haya anticipado recompensas cognitivas 0 afectivas, sino porque sería gratificante para ellos mismo.

\section{Inmersión estratégica}

Hace unas décadas, la profesión de la enseñanza de idiomas preocupaba en gran medida, debido a ello era importante sumarse a la entrega completa del lenguaje, en el caso del estudiante, proporcionando métodos, libros de texto, incluso paradigmas gramaticales, los cuales fueron citados como los factores esenciales en el éxito del aprendizaje y enseñanza del lenguaje (Brown,1994).

En años más recientes, a la luz de numerosos estudios sobre los estudiantes con y sin éxito, los profesores de idiomas están centrando más atención en el papel del estudiante en el proceso.

Los métodos que el estudiante emplea para internalizar y llevar a cabo en el idioma son tan importantes como los métodos del profesor o más aún, a esto se le llama el principio de la inversión estratégica.

El dominio de un segundo idioma se debe principalmente a la inversión personal de un estudiante que dedique tiempo, esfuerzo y atención a la segunda lengua en forma de una batería individualizada de estrategias para poder obtener la comprensión y producción del lenguaje.

\section{Principios afectivos en la enseñanza del inglés}

Con base en los planteamientos de Jack C. Richards y Theodore S. Rodgers (2001), los principios afectivos en la enseñanza del inglés están enfocados en el procesamiento emocional de los seres humanos, es desde esta perspectiva que se pueden apreciar los sentimientos de sí mismo, de las relaciones en una comunidad de estudiantes, y de los lazos afectivos entre el lenguaje y la cultura. Componente esencial para lograr la motivación de los estudiantes en el proceso formativo con relación a la enseñanza de las lenguas.

\section{Ego lenguaje}

El ego lenguaje se puede resumir en una enunciación muy reconocida, que refiere a como los seres humanos aprendemos a utilizar una segunda lengua. También es posible identificarla cuando se desarrolla un nuevo modo de pensar, sentir y actuar en la apropiación de una segunda lengua, es como adquirir una "segunda identidad". El nuevo "ego lenguaje", entrelazado con la segunda lengua, se puede crear fácilmente en el estudiante como una sensación de fragilidad, una actitud defensiva, y una elevación de las inhibiciones.
El principio del ego lenguaje también puede ser cariñosamente llamado el principio "borroso caliente", debido a que desde este principio, todos los estudiantes del idioma deben ser tratados con cuidado y cariño afectivo.

Este principio para el caso de los adultos, funciona de manera diferente, debido al nivel de desarrollo de su inteligencia ya que generalmente lo afectivo pasa a un segundo plano, por ello, este principio tiene mayor aplicabilidad en el aprendizaje de un segundo idioma con los niños en donde el rol de los profesores será ofrecer todo el apoyo afectivo que se pueda con ellos (Richards y Rodgers, 2001). Aunque esta perspectiva también puede tener aplicabilidad con los jóvenes estudiantes en la educación superior.

Los estudiantes sienten esta fragilidad, porque los arsenales estratégicos de sus egos nativos-basadas en el lenguaje, normalmente son bien desarrollados y resistentes al ataque. En algunos casos podemos observar el modelo del estudiante que se siente obsoleto, y tiene que valerse de su yo emocional con una batería lingüística que él mismo se siente mísero, y que se siente con una sensación de indefensión.

De acuerdo con H. Douglas Brown (1994) esta situación se puede contraer con algunas posibilidades como, por ejemplo: mostrar a los estudiantes una actitud abierta, en referencia a las técnicas y secuencias, las cuales tienen que ser más desafiantes cognitivamente, pero en el nivel afectivo.

\section{Confianza en sí mismo}

Otra manera de enunciar este principio es con la frase "puedo hacerlo", o con el principio de la auto-estima. En la enseñanza y en el aprendizaje es importante que cada persona sea capaz de crear su propia estrategia para poder realizar las diferentes actividades, mientras que la auto-confianza puede estar relacionada con el principio del ego lenguaje interior, más allá de destacar en la autoevaluación del estudiante y sin importar el grado de implicación del ego del idioma y que en pocas palabras, estaríamos hablando de la creencia de poder alcanzar totalmente las estrategias para que sean capaces de realizar las diferentes tareas, es lo que se conoce como éxito eventual (Dayan, 2015).

Algunas aplicaciones inmediatas que surgen en el aula, de acuerdo con este principio son: dar amplias garantías verbales y no verbales a los estudiantes, que les ayuden a escuchar a un profesor que afirma la creencia en la capacidad del estudiante, así como la secuencia de lo fácil a lo difícil; como profesor se necesita sostener la auto-confianza en lo que ya existe y poder construir donde no lo realizan. Asimismo, sus diferentes 
actividades en el aula, ocasionarían que se comenzarán a utilizar técnicas y conceptos más simples.

\section{Toma de riesgos}

De acuerdo con Richards y Rodgers (2001), el tercer principio afectivo interrelacionado con los dos últimos principios, es la importancia de que los estudiantes tomen riesgos calculados en el intento de usar el lenguaje, tanto de forma productiva y receptiva.

Los dos principios anteriores van a la par de las bases para la toma de riesgos, si los estudiantes reconocen su propia fragilidad del ego y optan por desarrollar la convicción de que sí pueden hacerlo, entonces están dispuestos a tomar esos riesgos necesarios, lo cual hará que estén listos para llevar a cabo cada una de las experiencias adquiridas para poder utilizarlas con fines significativos, hacer preguntas y hacer valer sus conocimientos.

Muchos contextos educativos en el mundo no fomentan la toma de riesgos, sino que animan a la corrección, la respuesta correcta y las "conjeturas" de retención, hasta que uno está seguro de ser corregido. En una gran cantidad de investigaciones educativas se muestra que en las aulas se refleja el principio de la toma de riesgos.

Por lo que existen algunos puntos que se deben tomar en cuenta, tal es el caso de crear un ambiente agradable en el aula, que anime a los estudiantes a practicar el idioma, a aventurar una respuesta, y no sólo esperar a que alguien más lo haga para ser voluntario de idiomas, además de poder presentar desafíos razonables en técnicas que no la hagan ni demasiado fácil ni demasiado difícil.

\section{Conexión de la lengua-cultura}

Douglas Brown (1994) menciona que la lengua y la cultura están íntimamente entrelazadas, en cualquier momento que se aprende con éxito un lenguaje, también se puede aprender algo de la cultura de los hablantes y por supuesto el lenguaje.

Uno de los aspectos de este principio se centra en la compleja interconexión entre la lengua y cultura. Cuando enseña un lenguaje, también se enseña un sistema complejo de costumbres culturales, valores y formas de pensar, sentir y actuar.

Alguna de las aplicaciones que se deben realizar en el aula, es la de discutir las diferencias entre las culturas con los estudiantes, haciendo hincapié en que ninguna cultura es mejor que otra, sino que el conocimiento de ellas es una faceta importante del aprendizaje del idioma. Es esencial incluir, dentro de las técnicas determinadas actividades o materiales, aquellos aspectos que pueden referir a la conexión entre la cultura y el lenguaje, con lo que podemos enseñar a los estudiantes las connotaciones culturales, especialmente en los aspectos sociolingüísticos de la lengua.

Un segundo aspecto de la conexión de la lengua-cultura es el grado en que sus estudiantes serán a su vez afectados por el proceso de aculturación, que variará con el contexto y los objetivos de aprendizaje. En muchos contextos, el segundo aprendizaje de idiomas, como ESL en los EE.UU., conlleva que los estudiantes se enfrenten a la realidad en toda regla de adaptación, a la vida en un país extranjero, completamente diferente con diversas emociones que acompañan las etapas de aculturación.

En el salón de clases se puede ayudar a los estudiantes a ser conscientes de la aculturación y sus etapas, así como destacar la importancia de la segunda lengua como una herramienta poderosa para el ajuste en la nueva cultura y ser especialmente sensibles a cualquier estudiante que aparente estar deprimido, y hacer lo que se pueda para poder ayudarlo.

Todos los referentes que se presentaron en los párrafos anteriores, servirán como base la interpretación e identificación de problemas detectados y su posible tratamiento en las eventualidades que promueven el abandono escolar en la Licenciatura en Idiomas.

\section{Construcción de objeto de investigación}

Con base en los antecedentes y los principios conceptuales que orientan la práctica de la enseñanza de lenguas, en este espacio presentamos parte de los resultados de un proyecto de investigación, que se planteó como pregunta principal ¿qué eventualidades intervienen, para que el estudiante de la Licenciatura en Idiomas de la División Académica de Educación y Artes (DAEA) opte por abandonar sus estudios? Para dar respuesta, se consideraron momentos de aproximación al objeto de estudio, en este caso, la elaboración de un diagnóstico sobre las percepciones que tienen los estudiantes del segundo semestre de la carrera sobre ciertas eventualidades que pudieran estar influyendo en su permanencia o abandono de la Licenciatura.

Lo que nos llevó a definir, como objetivo general, Identificar, mediante un diagnóstico, qué eventualidades podrían estar influyendo en la permanencia o abandono de la carrera, para que a partir de su determinación se puedan diseñar estrategias que consideren los estilos de aprendizaje, las experiencias académicas y las condiciones institucionales de la Licenciatura en Idiomas. Para dar respuesta a tal interrogante, a continuación se describe el enfoque metodológico que se utilizó en el desarrollo de la investigación. 


\section{Enfoque metodológico}

La presente investigación se llevó a cabo bajo un enfoque cuantitativo de tipo explicativo (Hernández, Fernández y Baptista, 2010), donde se identifican las percepciones de los estudiantes de la Licenciatura en Idiomas en la División Académica de Educación y Artes de la Universidad Juárez Autónoma de Tabasco sobre los elementos que pudieran estar influyen en la posibilidad de abandono escolar en esta carrera.

\section{Contexto y diseño de la muestra}

La realización de esta investigación se llevó a cabo con una muestra de 30 estudiantes, de un universo de 120 estudiantes del turno vespertino de dicha Licenciatura, ubicada en la Colonia Magisterial de la Ciudad de Villahermosa, en el Estado de Tabasco.

Respecto a las características de la población objetivo, el rango promedio de edad oscila entre los 19 y 21 años, en donde la distribución por género es de 70 hombres y 50 mujeres.

La mayoría de estudiantes provienen de comunidades (municipios) que se dedican la agricultura y la fabricación de artesanías, y ellos ayudan en dichas actividades para su sustento. Es importante mencionar que son pocos los estudiantes que cuentan con un trabajo formal remunerado, y son ellos quienes se sostienen económicamente (estudiantes independientes). Los que cuentan con padres con un buen empleo sólo se dedican a estudiar. Respecto al deber ser sobre la preparación del personal docente, deben contar, de preferencia, con un grado de maestría o doctorado, para poder impartir clases en esta universidad.

\section{Instrumento}

El instrumento aplicado fue un cuestionario que consta de 14 preguntas a escala tipo Likert, en donde todas son de opción múltiple. Las primeras dos preguntas, tienen como objetivo, conocer sobre la experiencia que tuvieron los estudiantes en el bachillerato, y si tenían nociones del Idioma Inglés, antes de ingresar al nivel Universitario.

La materia de inglés tiene un gran peso en la Licenciatura en Idiomas, por ello nos interesó saber si los estudiantes son vulnerables a reprobar estas materias. De la misma forma, interesó conocer si los estudiantes dan de baja muchas materias y cuáles en específico.

\section{Resultados}

1. Rendimiento académico en las asignaturas de inglés durante el bachillerato.
El nivel medio superior, como antecedente formativo, es un factor que hay que tener en cuenta, ya que constituye la base para identificar porque los estudiantes tienen 0 no éxito, en este caso sobre el dominio del idioma inglés. Respecto al rendimiento e interés por el habla inglesa, se encontró que $75 \%$ de los estudiantes (23) reportó que su rendimiento ha sido bueno, mientras que el $25 \%$ restante (7 estudiantes) menciona que su rendimiento fue regular, tal como se muestra en el siguiente gráfico:

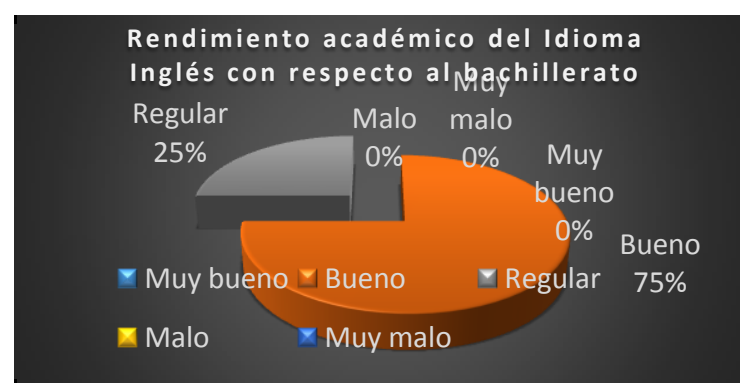

Figura 1. Rendimiento académico en el idioma inglés durante el bachillerato

2. Motivos del bajo desempeño académico en el bachillerato

El bachillerato es un filtro importante para poder decidir hacia dónde se inclina el estudiante. Al mismo tiempo, es un referente sobre las diferentes causas que dan origen a un bajo desempeño en este nivel. En tal sentido y como lo muestra el gráfico de la figura 2, $50 \%$ de los estudiantes encuestados afirma que la falta de retención de los idiomas es una causa que provoca bajo rendimiento, mientras que $38 \%$ lo atribuye a la falta de didáctica por parte de los profesores. Finalmente, 12\% menciona la falta de impulso de los padres hacia los hijos, que puede referirse a diversos tipos de apoyos de los padres a los estudiantes en este nivel.

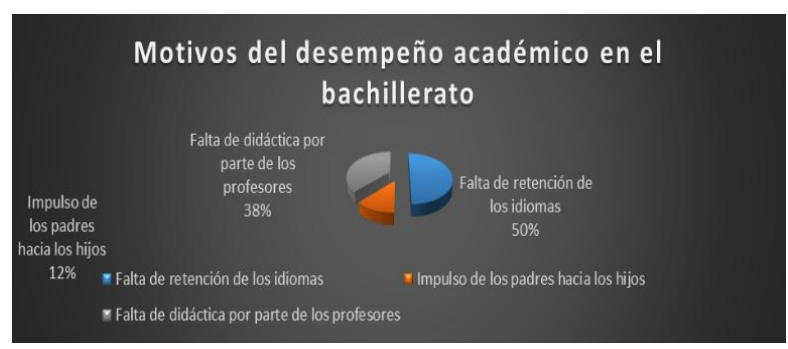

Figura 2. Motivos sobre el bajo desempeño en bachillerato.

3. Conocimiento del idioma inglés antes de ingresar al nivel universitario

Otro factor importante al momento de ingresar a la licenciatura en idiomas es tener pocos conocimientos previos del idioma inglés, sobre los cual encontramos que $20 \%$ de la población estudiada afirma conocer mucho el idioma y $30 \%$ bastante, lo cual indica que la mitad de la muestra considera tener alto conocimiento 
del idioma antes de ingresar a la universidad, mientras que $23 \%$ refiere que lo conocía medianamente y $20 \%$ poco. Solamente $7 \%$ afirma que no contaba con nada de conocimiento sobre el idioma inglés antes de ingresar a la Licenciatura. Por lo que se esperaría que el índice de abandono en esta licenciatura fuera similar o menor al $7 \%$ o por lo menos que no rebasara el $20 \%$, considerando que el poco conocimiento también podría llevar a los estudiantes a dejar el programa.

\begin{tabular}{|c|c|c|}
\hline \multicolumn{3}{|c|}{$\begin{array}{l}\text { 3.- ¿Cuándo ingresaste al nivel universitario tenías conocimiento del Idioma } \\
\text { Inglés? }\end{array}$} \\
\hline & FRECUENCIA & PORCENTAJE \\
\hline Mucho & 6 & $20 \%$ \\
\hline Bastante & 9 & $30 \%$ \\
\hline Medianamente & 7 & $23 \%$ \\
\hline Poco & 6 & $20 \%$ \\
\hline Nada & 2 & $7 \%$ \\
\hline Muy poco & 0 & $0 \%$ \\
\hline
\end{tabular}

Figura. 3 Tabla de distribución de frecuencias sobre el dominio del inglés previo al ingreso a la licenciatura

\section{Fuente de financiamiento de los estudios}

Es común hoy en día, que el abandono escolar sea atribuible al tipo de apoyo financiero que reciben los estudiantes en su periodo educativos. No obstante, de acuerdo los resultados obtenidos en el caso de los estudiantes encuestados, se encontró que la principal fuente de financiación de los estudios, para la mitad de ellos corresponde a la ayuda de sus padres y, para la otra mitad al trabajo propio.

\section{Experiencia o el contacto con los idiomas}

Haber tenido alguna experiencia con los idiomas tiene un gran significado, debido a que es ahí donde se puede identificar el interés de los estudiantes en este rubro, para con ello, poder pre-determinar el rendimiento académico que podría tener en la licenciatura. Es este sentido, 38\% de la población representada menciona que ha tenido mala la experiencia con los idiomas, aunado al $13 \%$ que alude a que ha sido pésima y al $12 \%$ que la considera regular, que indica que la mayoría ha tenido experiencias desagradable respecto a los idiomas, mientras que sólo una proporción menor (37\%) refirió haber tenido buena experiencia con los idiomas.

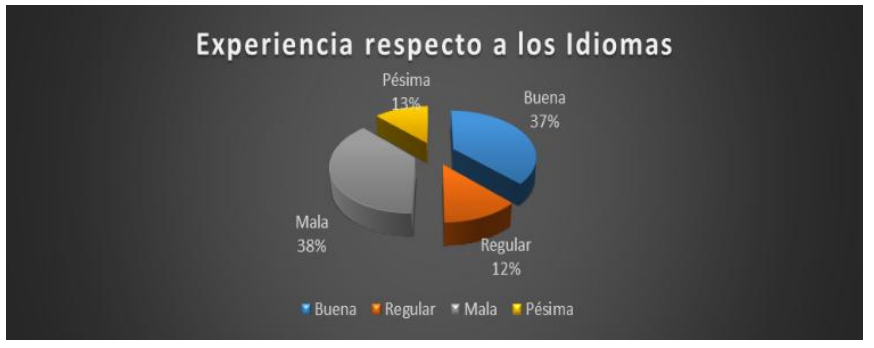

Figura 4. Experiencia o el contacto con los idiomas

6. Índice de reprobación del idioma inglés en la universidad

Como se aprecia en el gráfico 5 , sobre el índice de reprobación de las materias del idioma inglés en la licenciatura, la mayoría (63\%) no ha reprobado materias del área inglesa, por lo que se podría deducir que el 37\% restante, podría requerir apoyo socio-afectivo y cognitivo, por el hecho de haber reprobado materias de esta área.

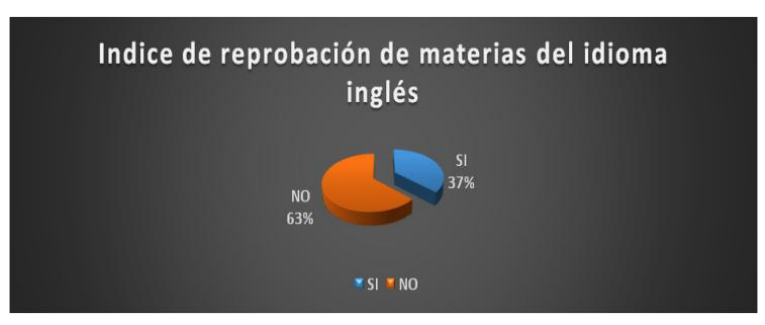

Figura 5. Índice de reprobación materias del idioma inglés en la Licenciatura

7. Distribución de frecuencias en la baja de materias de licenciatura

Dar de baja a alguna materia, es un proceso que se puede llevar a cabo en el nivel universitario, y por ello consideramos importante sondear al respecto, ya que esto da margen para compensar los rendimientos en licenciatura. Se identificó (ver tabla 6) que no hay un comportamiento regular en esta actividad. No obstante, el mayor porcentaje (27\%) que da de baja materias se ubica en hacerlo medianamente, seguido del $20 \%$ que lo hace bastante o muy poco (20\%). Por ello, esta actividad resulta poco significativa para poder determinar si influye en la posibilidad de abandono escolar por parte de los estudiantes. 


\begin{tabular}{|l|l|l|}
\hline \multicolumn{2}{|c|}{ 7.- ¿Con qué frecuencia le das de baja a una o más materias? } \\
\hline & FRECUENCIA & PORCENTAJE \\
\hline Mucho & 4 & $13 \%$ \\
\hline Bastante & 6 & $20 \%$ \\
\hline Medianamente & 8 & $27 \%$ \\
\hline Poco & 3 & $10 \%$ \\
\hline Nada & 3 & $10 \%$ \\
\hline Muy poco & 6 & $20 \%$ \\
\hline
\end{tabular}

Figura.6. Frecuencias sobre baja de materias en licenciatura

Otro aspecto que resultó poco relevante, refiere a las experiencias negativas que han tenido los estudiantes con sus profesores, ya que la mitad dice que sí y la otra que no.

\section{Cambio de carrera}

En cuanto a los cambios de carrera, solo $37 \%$ los ha hecho, por lo que se considera que la mayoría (63\%) tiene una permanencia continua con sus estudios. Lo que de alguna manera refleja cierta estabilidad en la matrícula de la Licenciatura.

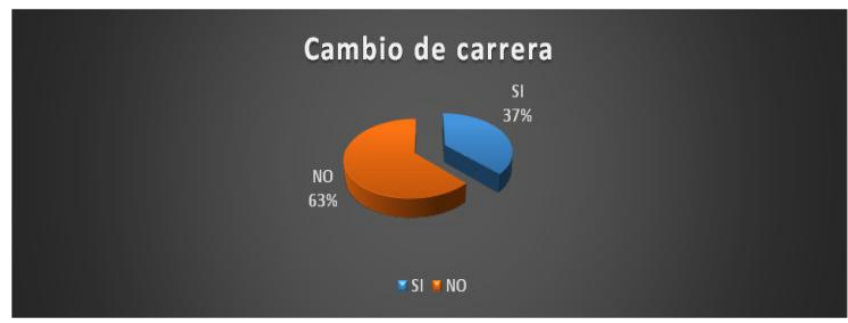

Figura 7. Distribución por cambio de carrera

\section{Disposición para estudiar inglés}

Dado que se trata de identificar qué tanta disposición tienen los estuantes con relación al interés por este tipo de carrera, la actitud para la dedicación al dominio del inglés resulta muy relevante, por lo que se encontró que más de la mitad (58\%) de los estudiantes ponen mucho de su parte para estudiar el idioma inglés, seguido del $23 \%$ que los hace bastante. El resto de la muestra lo hace medianamente $(10 \%)$ o poco $(9 \%)$, pero lo hace, ya que ninguno reportó no dedicarse.

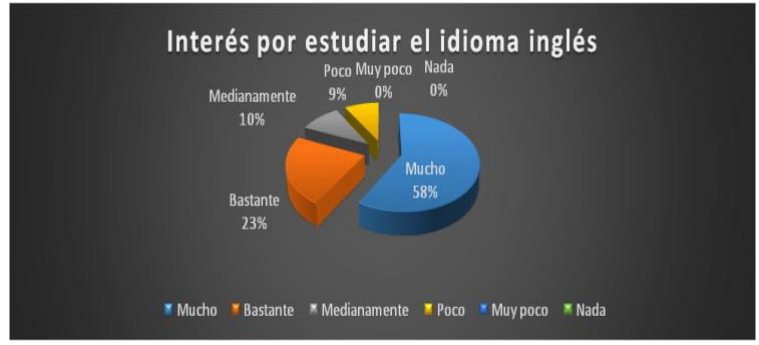

Figura 8. Disposición para estudiar inglés

La disposición para el aprendizaje del inglés también tiene relación con los estilos de aprendizaje, ya que la mayoría de los estudiantes consideran que éstos influyen bastante $(47 \%)$, medianamente $(30 \%)$ o mucho $(17 \%)$ en la excelencia de los resultados.

\section{Uso de los estilos de aprendizaje}

La aplicación de los estilos cognitivos (formas o estilos de aprendizaje) es muy importante, debido a que dan como resultado una excelente respuesta para el logro educativo, mediante su uso se aprende más rápido, en No obstante, menos de la mitad (47\%) de los estudiantes los aplica medianamente y una cantidad considerable lo hace bastante (30\%) o mucho (10\%).ión Lama la atención que haya quienes los apliquen poco (6\%) o nada $(7 \%)$ puesto que para ellos dicha importancia pasa desapercibida.

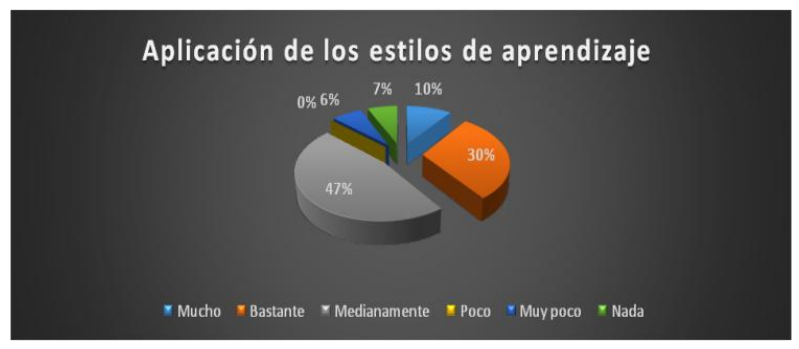

Figura. 9. Aplicación de los estilos de aprendizaje

11. Materiales de apoyo para el buen funcionamiento del programa

Cualquier programa educativo debe de contar con los materiales necesarios para poder proporcionar al estudiante las mejores condiciones de aprendizaje, lo cual es notorio en la percepción de los estudiantes, ya que la gran mayoría (44\% y 43) identifican que se cuenta bastante con ellos o medianamente, respectivamente. $\mathrm{Si}$ 
bien la proporción que considera que el programa cuento con muy pocos (10\%) materiales o poco (3\%) es mínima, se debe tomar como un aspecto de reflexión, será necesario poner mayor énfasis en la producción de éstos.

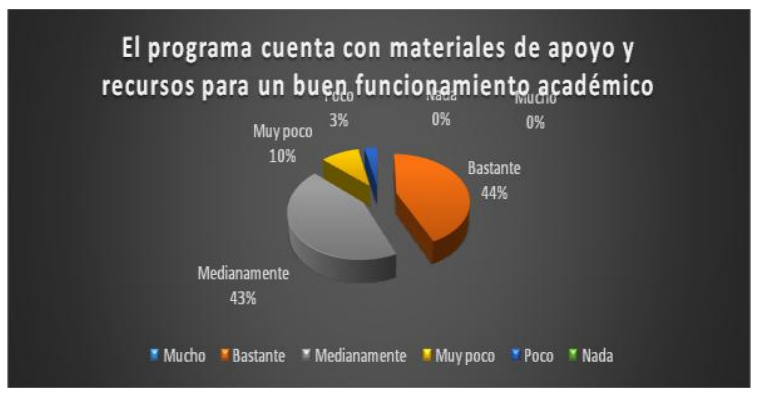

Figura 10. Identificación de materiales de apoyo y recursos para el buen funcionamiento del programa

12. Evaluación continua para apoyo a los estudiantes

La percepción de los estudiantes respecto a la presencia de la evaluación continua para apoyar su aprendizajes está muy identificada en los estudiantes, ya que la mayoría menciona que se realiza bastante (44\%), mucho ((30\%) o medianamente (23\%). Sólo 3\% reconoce que se realiza muy poco. En este sentido es importante reconocer que aunque existe la presencia de la evaluación para mejorar la calidad educativa, también es importante reconocer que existen otros mecanismos (auto-evaluación y co-evaluación) de evaluación que podrían aportar mayores elementos para el incremento de dicha calidad.

\begin{tabular}{|l|l|l|}
\hline \multicolumn{3}{|c|}{$\begin{array}{l}\text { 14.- ¿Se realizan evaluaciones constantes para detectar cómo van los } \\
\text { estudiantes y así prestarles ayuda? }\end{array}$} \\
\hline & FRECUENCIA & PORCENTAJE \\
\hline Mucho & 9 & $30 \%$ \\
\hline Bastante & 13 & $44 \%$ \\
\hline Medianamente & 7 & $23 \%$ \\
\hline Poco & 0 & $0 \%$ \\
\hline Nada & 0 & $0 \%$ \\
\hline Muy poco & 1 & $3 \%$ \\
\hline
\end{tabular}

Figura 11. Tabla Evaluación continua para apoyo a los estudiantes

\section{Conclusiones}

De acuerdo con los resultados, se considera que se cuenta con elementos de base para la conformación de un diagnóstico respecto a las percepciones que tienen los estudiantes del segundo semestre de la Licenciatura en Idiomas, sobre las eventualidades que pudieran estar interviniendo en el abandono y rendimiento escolar de los estudiantes. Así, con relación a su experiencia en el bachillerato, la mayoría tuvo buen rendimiento, además de que la mitad tenía conocimiento del idioma antes de ingresar a licenciatura, pero este caso llama la atención que la mitad de los estudiantes atribuyan los motivos de bajo rendimiento a su falta de retención y otros tantos, a la mala didáctica del docente.

En cuanto a su financiamiento para permanecer en la carrera, sería conveniente dar seguimiento al grupo, ya que la mitad lo hace por cuenta propia y la otra mitad, con apoyo de sus padres, por lo que habría que identificar quiénes presentan mayor posibilidad de permanencia.

Por otro lado, aunque se identificó que poco más de la mitad han tenido malas experiencias relacionadas con el idioma, la mayoría no ha reprobado las materias relacionadas, ni tampoco dan de baja materias de manera frecuente. Esto aunado a que la permanencia de los estudiantes en la carrera es de más del sesenta por ciento, lo que refleja estabilidad en quienes se encuentran en ella durante estos dos primeros semestres.

Quizá por ser la parte inicial del proyecto formativo, los estudiantes declararon tener disposición para el aprendizaje del idioma inglés, no obstante, la aplicación de los estilos de aprendizaje para ello parece no ser tan importante, dado que no todos se ocupan de hacerlo.

Finalmente, los estudiantes identificaron dos condiciones importantes para alcanzar logros significativos en el desarrollo de esta carrera, uno es sobre la existencia de materiales y recursos de apoyo y el otro, sobre la existencia de la evaluación continúa como referente para atender los problemas de rendimiento en los estudiantes.

Esta información constituye el punto de partida para establecer correlaciones entre los indicadores y de ahí articular los resultados con los principios para el aprendizaje y, de acuerdo con ellos, desarrollar los modelos y métodos de enseñanza acordes a las necesidades. 


\section{Referencias bibliográficas}

Brown H. Douglas. (1994). Teaching by principles an interactive approach language pedagogy. London: Longman. Disponible en: https://octovany.files.wordpress.com/2013/12/ok-teaching-byprinciples-h-douglas-brown.pdf (Consultado 16 de diciembre, 2018).

Carrasco, José Bernardo y Basterretche, Juan (2004) Técnicas y recursos para motivar a los estudiantes, México: Ediciones Rialp.

Hernández, R. Fernández y Baptista, (2010). Metodología de la investigación, 4ta edición. México: Mc Graw Hill.

Richards, Jack C. y Rodgers, Theodore S. (2001) Approaches and Methods in Language Teaching. Cambridge Language Teaching Library. Disponible en: https://www.novaconcursos.com.br/blog/pdf/richardsjack-c.-\&-rodgers.pdf (Consultado 20 de febrero, 2019).

Titone, Renzo (1968). Teaching Foreign Languages: An Historical Sketch. Georgetown University Press. 